\title{
ORDERED CYCLE LENGTHS IN A RANDOM PERMUTATION
}

\author{
BY \\ L. A. SHEPP AND S. P. LLOYD
}

1. Introduction. Problems involving a random permutation are often concerned with the cycle structure of the permutation. Let $\mathscr{S}_{n}$ be the $n$ ! permutation operators on $n$ numbered places, and let $\alpha(\pi)=\left(\alpha_{1}(\pi), \alpha_{2}(\pi), \cdots, \alpha_{n}(\pi)\right)$ designate the cycle class of $\pi \in \mathscr{S}_{n}$; that is, permutation $\pi$ has $\alpha_{1}(\pi)$ cycles of length $1, \alpha_{2}(\pi)$ cycles of length $2, \cdots$. Suppose the elements of $\mathscr{S}_{n}$ are assigned probability $1 / n$ ! each. In a variety of problems one seeks limiting (large $n$ ) properties of random variables which depend on $\pi$ only by way of $\alpha(\pi)$. In the matching problem, for instance, the result $\lim _{n}$ prob $\left\{\alpha_{1}=0\right\}=e^{-1}$ is an old one [1, p. 50]. Goncharov in [2], [3] gives limiting forms for the distribution of each $\alpha_{j}$, of $\sum \alpha_{j}$, and of the longest cycle. In [4], [5] Golomb also investigates the longest cycle. With $l_{n}$ the expected length of the longest cycle, Golomb shows that $l_{n} / n$ is monotone decreasing, and gives the numerical value $0.62432965 \cdots$ for the limit. Answering in part a question raised by Golomb, we give a closed form for this limit (Equation (14)); in fact, we give in $\S 4$ the corresponding result for the $m$ th moment of the length of the $r$ th longest cycle for $m=1,2, \cdots$ and $r=1,2, \cdots$, and we give the limiting distribution for the length of the $r$ th longest cycle. In $\$ 5$ we give asymptotics for the distribution and all moments of the length of the $r$ th shortest cycle, $r=1,2, \cdots$. The results and proofs are more complicated for the moments of the $r$ th shortest cycle than for the $r$ th longest. Our methods are straightforward: we set up generating functions, get leading terms in closed form, and use Tauberian methods to recover the asymptotic dependence on $n$. The Tauberian side conditions needed are based on the combinatorial arguments of $\S 6$.

2. A model. For given $n$ the distribution of $\alpha$ is obtained by dividing the number of permutations in class $\alpha$ by $n$ !:

$$
\begin{aligned}
P\left\{\alpha_{1}\right. & \left.=a_{1}, \alpha_{2}=a_{2}, \cdots, \alpha_{n}=a_{n}\right\} \\
& =\prod_{j=1}^{n} \frac{(1 / j)^{a_{j}}}{a_{j} !} \text { if } \sum_{j=1}^{n} j a_{j}=n, \\
& =0 \quad \text { otherwise, }
\end{aligned}
$$

the $a$ 's being any nonnegative integers $[1, \mathrm{p} .67]$. It will prove natural to include

Presented to the Society, September 2, 1965 ; received by the edittors April 7, 1965. 
the case $n=0 ; \mathscr{S}_{0}$ consists of 0 ! elements, namely, the identity permutation on the empty set (all $\alpha$ 's are 0 ).

The $\alpha$ 's above would be independent if it were not for the condition on $\sum j a_{j}$. Consider then a sequence $\alpha=\left(\alpha_{1}, \alpha_{2}, \cdots\right)$ of mutually independent nonnegative integer valued random variables, where for $j=1,2, \cdots$ the random variable $\alpha_{j}$ has the Poisson distribution of mean $z^{j} / j$, viz.:

$$
P_{z}\left\{\alpha_{j}=a\right\}=\exp \left[-\left(z^{j} / j\right)\right] \frac{\left(z^{j} / j\right)^{a}}{a !}, \quad a=0,1, \cdots
$$

Here, the parameter $z$ which has been introduced is such that $0<z<1$ and is the same for each $j=1,2, \cdots$. From now on, probabilities and expectation based on the family (2) will carry subscript $z$, while those based on (1) will carry subscript $n$.

We have $P_{z}\left\{\alpha_{j} \neq 0\right\}=1-\exp \left[-z^{j} / j\right]<z^{j} / j$, so that $\sum_{j=1}^{\infty} P_{z}\left\{\alpha_{j} \neq 0\right\}$ is finite. By the Borel-Cantelli lemma, $P_{z}\left\{\alpha_{j} \neq 0\right.$ for infinitely many $\left.j\right\}=0$. Thus the random variable $v(\alpha)=\sum_{j=1}^{\infty} j \alpha_{j}$ is finite with probability 1 , and the joint distribution of the $\alpha$ 's may be written meaningfully as

$$
P_{z}\left\{\alpha_{1}=a_{1}, \alpha_{2}=a_{2}, \cdots\right\}=(1-z) z^{v(a)} \prod_{j=1}^{\infty} \frac{(1 / j)^{a_{j}}}{a_{j} !}
$$

for all sequences $a=\left(a_{1}, a_{2}, \cdots\right)$ of nonnegative integers eventually 0 . It is easy to see that the conditional distribution of the $\alpha$ 's given $v$ does not depend on $z$; it is just

$$
\begin{aligned}
P_{z}\left\{\alpha_{1}\right. & \left.=a_{1}, \alpha_{2}=a_{2}, \cdots \mid v(\alpha)=n\right\} \\
& =\prod_{j=1}^{n} \frac{(1 / j)^{a_{j}}}{a_{j} !} \text { if } \sum_{j=1}^{\infty} j a_{j}=n, \\
& =0 \quad \text { otherwise, }
\end{aligned}
$$

so that we have recovered (1). On the other hand, the distribution of $v$ is $P_{z}\{v(\alpha)=n\}=(1-z) z^{n}, n=0,1, \cdots$; the degree of a random permutation under $P_{z}$ is a geometrically distributed random variable. Its expected value is $E_{z}\{v\}=z /(1-z)$, so that $z \rightarrow 1$ will correspond to $n \rightarrow \infty$.

Suppose $\Phi=\Phi(\alpha)$ is some given functional of the cycle class $\alpha=\left(\alpha_{1}, \alpha_{2}, \cdots\right)$ of a random permutation, defined for all degrees; we put $\alpha_{n+1}(\pi)=\alpha_{n+2}(\pi)=\cdots=0$ if $\pi \in \mathscr{S}_{n}$. By what has been said above,

$$
\begin{aligned}
E_{z}\{\Phi\} & =E_{z}\left\{E_{z}\{\Phi \mid v\}\right\} \\
& =\sum_{n=0}^{\infty} P_{z}\{v=n\} E_{z}\{\Phi \mid v=n\} \\
& =\sum_{n=0}^{\infty}(1-z) z^{n} E_{n}\{\Phi\}
\end{aligned}
$$


where $E_{n}(\Phi)$ is the expected value of $\Phi$ for the distribution (1). Thus $E_{z}(\Phi) /(1-z)$ is the generating function for the sequence $E_{n}\{\Phi\}, n=0,1, \cdots$.

To illustrate, we derive the limiting distributions of each $\alpha_{j}$ and $\sum \alpha_{j}$ obtained by Goncharov. Under $P_{z}, \alpha_{j}$ is Poisson with mean $z^{j} / j$, so the characteristic function of $\alpha_{j}$ has the familiar form

$$
E_{z}\left\{\exp \left[i t \alpha_{j}\right]\right\}=\exp \left[\left(z^{j} / j\right)\left(e^{i t}-1\right)\right], \quad-\infty<t<\infty .
$$

Using our basic relation (3), it is straightforward that

$$
\begin{aligned}
E_{n}\left\{\exp \left[i t \alpha_{j}\right]\right\} & =\sum_{p=0}^{p \leqq n / j} \frac{1}{p !}\left(\frac{e^{i t}-1}{j}\right)^{p}, \\
\lim _{n \rightarrow \infty} E_{n}\left\{\exp \left[i t \alpha_{j}\right]\right\} & =\exp \left[(1 / j)\left(e^{i t}-1\right)\right], \quad-\infty<t<\infty .
\end{aligned}
$$

This last is the characteristic function of the Poisson distribution of mean $1 / j$. Since $P_{n}\left\{\alpha_{j}\right.$ is an integer $\}=1$, the theorem of Paul Lévy gives

$$
\lim _{n \rightarrow \infty} P_{n}\left\{\alpha_{j}=a\right\}=\exp [-(1 / j)] \frac{(1 / j)^{a}}{a !}, \quad a=0,1, \cdots,
$$

for the limiting distribution of $\alpha_{j}$. Consider next the total number of cycles $\sigma(\alpha)=\sum_{1}^{\infty} \alpha_{j}$. Under $P_{z}$, the $\alpha$ 's are Poisson and independent, so $\sigma$ is Poisson with mean $\sum_{1}^{\infty}\left(z^{j} / j\right)=\log (1 /(1-z))$. Hence

$$
E_{z}\left\{e^{i t \sigma}\right\}=\left[\frac{1}{1-z}\right]^{(\exp [i t]-1)}, \quad-\infty<t<\infty,
$$

and the coefficient of $z^{n}$ in $E_{z}\left\{e^{i t \sigma}\right\} /(1-z)$ is the binomial coefficient

$$
E_{n}\left\{e^{i t \sigma}\right\}=\frac{\Gamma\left(n+e^{i t}\right)}{\Gamma\left(e^{i t}\right) n !}, \quad-\infty<t<\infty, \quad n=0,1, \cdots .
$$

Using a Binet form of the Stirling approximation [6, p. 249] we obtain easily

$$
E_{n}\left\{e^{i t \sigma}\right\}=\frac{n^{(\exp [i t]-1)}}{\Gamma\left(e^{i t}\right)}\left[1+\frac{\theta_{n}(t)}{n-1}\right], \quad-\infty<t<\infty, \quad n=2,3, \cdots,
$$

with $\left|\theta_{n}(t)\right| \leqq M<\infty$ uniformly in the range indicated. The mean and variance of $\sigma$ under $P_{n}$ are

$$
\begin{aligned}
E_{n}\{\sigma\} & =\sum_{p=1}^{n} \frac{1}{p}=\log n+O(1) \\
E_{n}\left\{\sigma^{2}\right\}-E_{n}^{2}\{\sigma\} & =\sum_{p=1}^{n} \frac{p-1}{p^{2}}=\log n+O(1),
\end{aligned}
$$

and for the normalized variable $(\sigma-\log n) / \sqrt{ } \log n$ we obtain from (4) 


$$
\lim _{n \rightarrow \infty} E_{n}\left\{\exp i t\left(\frac{\sigma-\log n}{\sqrt{ } \log n}\right)\right\}=\exp \left[-\frac{1}{2} t^{2}\right], \quad-\infty<t<\infty .
$$

We apply the Lévy theorem and have Goncharov's result

$$
\lim _{n \rightarrow \infty} P_{n}\left\{\frac{\sigma-\log n}{\sqrt{ } \log n} \leqq x\right\}=\int_{-\infty}^{x} \exp \left[-\frac{1}{2} u^{2}\right] \frac{d u}{\sqrt{ } 2 \pi}, \quad-\infty<x<\infty .
$$

In the same vein, let $\sigma^{\prime}=\alpha_{2}+\alpha_{4}+\cdots$ be the total number of odd cycles. (That is, $(-1)^{\sigma^{\prime}}$ is the character of the alternating representation.) Just as above, we obtain

$$
\begin{aligned}
E_{z}\left\{e^{i t \sigma^{\prime}}\right\} & =\left[\frac{1}{1-z^{2}}\right]^{(\exp [i t]-1) / 2}, \\
E_{n}\left\{e^{i t \sigma^{\prime}}\right\} & =\frac{\Gamma\left(\frac{1}{2}\left(e^{i t}+1\right)+p\right)}{\Gamma\left(\frac{1}{2}\left(e^{i t}+1\right)\right) p !}, \quad-\infty<t<\infty \\
n & =2 p \text { or } 2 p+1, \\
p & =0,1, \cdots,
\end{aligned}
$$

and $\left(\sigma^{\prime}-\frac{1}{2} \log n\right) / \sqrt{ }\left(\frac{1}{2} \log n\right)$ is standard Gaussian in the limit.

3. The model extended. The following setting for the probability measure $P_{z}$ will render transparent the formulas for generating functions involving the $r$ th longest and $r$ th shortest cycles. Let a Poisson process take place on $T=\{-\infty<t<\infty\}$ at unit rate. That is, if $I \subset T$ is any interval of length $|I|$, the probability that $p$ jumps of the process occur in $I$ is $e^{-|I|}|I|^{p} / p !, p=0,1, \cdots$, independently of any conditions on the process on $T-I$. We lay off to the right of the origin successive intervals of length $z^{j} / j, j=1,2, \cdots$. Explicitly, the endpoints are

$$
\begin{aligned}
t_{1}(z) & =0 \\
t_{2}(z) & =\frac{z}{1}, \\
t_{3}(z) & =\frac{z}{1}+\frac{z^{2}}{2}, \\
& \vdots \\
t_{j}(z) & =\sum_{1}^{j-1} \frac{z^{k}}{k}, \\
& \vdots \\
t_{\infty}(z) & =\sum_{1}^{\infty} \frac{z^{k}}{k}=\log \frac{1}{1-z},
\end{aligned}
$$


and the $j$ th interval is $\left\{t: t_{j}(z) \leqq t<t_{j+1}(z)\right\}, j=1,2, \cdots$. Let $\lambda_{z}(t),-\infty<t<\infty$, be the function whose value is $j$ on the $j$ th interval, $j=1,2, \cdots$, and 0 if $t<0$ or $t \geqq t_{\infty}(z)$. Then for each $j=1,2, \cdots$ the interval $\left\{t: \lambda_{z}(t)=j\right\}$ has length $z^{j} / j$, the probability that $a_{j}$ jumps of the Poisson process occur in this interval is $\exp \left[-\left(z^{j} / j\right)\right]\left(z^{j} / j\right)^{a_{j}} / a_{j} !, a_{j}=0,1, \cdots$, and these various events for $j=1,2, \cdots$ are mutually independent; we are back to $P_{z}$. To be quite explicit, here is how we may choose a random permutation according to $P_{z}$. We take a sample function of the Poisson process; the jumps in the interval $\left[0, t_{\infty}(z)\right)$ are finite in number with probability 1 , occurring at the times $\tau_{1} \leqq \tau_{2} \leqq \cdots \leqq \tau_{\sigma}$, say (with $\sigma$ random, of course). We take the positive integers $\lambda_{z}\left(\tau_{1}\right) \leqq \lambda_{z}\left(\tau_{2}\right) \leqq \cdots \leqq \lambda_{z}\left(\tau_{\sigma}\right)$ as the lengths of the $\sigma$ cycles of a permutation on $v=\sum_{s=1}^{\sigma} \lambda_{z}\left(\tau_{s}\right)$ places, and in this class of $\mathscr{S}_{v}$ we choose a permutation at random with uniform distribution.

We will see now that this machinery is well adapted to investigating the $r$ th longest and $r$ th shortest cycles for any given $r=1,2, \cdots$. Let $S_{r}=S_{r}(\alpha)$ be the length of the $r$ th shortest cycle in a permutation of cycle class $\alpha$; we define $S_{r}(\alpha)=0$ if $\sum \alpha_{j}<r$. The probability density at $t$ of the $r$ th jump of the Poisson process counting to the right from $t_{1}(z)$ is $t^{r-1} e^{-t} /(r-1) !, 0 \leqq t<\infty$, as is well known. If this $r$ th jump occurs at $t$, then the value of $S_{r}$ is $\lambda_{z}(t)$, according to our model. It follows that the distribution of $S_{r}$ under $P_{z}$ is

$$
\begin{aligned}
P_{z}\left\{S_{r}=j\right\} & =\int_{t_{j}(z)}^{t_{j+1}(z)} \frac{t^{r-1}}{(r-1) !} e^{-t} d t, \quad j=1,2, \cdots, \\
& =\int_{t_{\infty}(z)}^{\infty} \frac{t^{r-1}}{(r-1) !} e^{-t} d t, \quad j=0 .
\end{aligned}
$$

We will treat this in more detail in $\$ 5$.

Let $L_{r}=L_{r}(\alpha)$ be the length of the $r$ th longest cycle in a permutation of cycle class $\alpha$; we define $L_{r}(\alpha)=0$ if $\sum \alpha_{j}<r$. The probability density at $t$ of the $r$ th jump of the Poisson process counting to the left from $t_{\infty}(z)$ is

$$
\left[t_{\infty}(z)-t\right]^{r-1} \exp \left[-\left[t_{\infty}(z)-t\right]\right] /(r-1) !, \quad-\infty<t \leqq t_{\infty}(z) .
$$

As with (5) then,

$$
\begin{aligned}
P_{z}\left\{L_{r}=j\right\} & =\int_{t_{j}(z)}^{t_{j+1}(z)} \frac{\left[t_{\infty}(z)-t\right]^{r-1}}{(r-1) !} \exp \left[-\left[t_{\infty}(z)-t\right]\right] d t, \quad j=1,2, \cdots \\
& =\int_{-\infty}^{0} \frac{\left[t_{\infty}(z)-t\right]^{r-1}}{(r-1) !} \exp \left[-\left[t_{\infty}(z)-t\right]\right] d t, \quad j=0 .
\end{aligned}
$$

We consider this in detail in the next section.

We conclude the present section with some analytical preliminaries regarding the $t_{j}(z)$. With $z=e^{-s}, 0<s<\infty$, we have 


$$
t_{\infty}\left(e^{-s}\right)-t_{j}\left(e^{-s}\right)=\sum_{j}^{\infty} \frac{e^{-k s}}{k}, \quad j=1,2, \cdots .
$$

On the interval $\{y: k s<y<(k+1) s\}$ there obtains

and

$$
\frac{e^{-k s}}{k s}>\frac{e^{-y}}{y}>\frac{e^{-(k+1) s}}{(k+1) s}
$$

$$
\frac{e^{-k s}}{k}>\int_{k s}^{(k+1) s} \frac{e^{-y}}{y} d y>\frac{e^{-(k+1) s}}{k+1} .
$$

Summing on $k$, we have bounds

$$
E(j s)<\sum_{j}^{\infty} \frac{e^{-k s}}{k}<E((j-1) s), \quad j=1,2, \cdots,
$$

where

$$
E(x)=\int_{x}^{\infty} \frac{e^{-y}}{y} d y, \quad 0<x<\infty, \quad(=+\infty, x=0),
$$

is the exponential integral. The mapping $x \rightarrow E(x)$ is an order reversing homeomorphism of $0<x<\infty$ onto itself, so for each $j=1,2, \cdots$ and each $0<s<\infty$ the equation

$$
\sum_{j}^{\infty} \frac{e^{-k s}}{k}=E\left(x_{j}(s)\right)
$$

has a unique root $0<x_{j}(s)<\infty$. Using (7), we obtain $(j-1) s<x_{j}(s)<j s$, $j=1,2, \cdots$.

In $\S 5$ we will need a closer estimate for $x_{1}(s)$. From the well-known identity

$$
E(x)=\int_{0}^{x} \frac{1-e^{-y}}{y} d y-\log x-\gamma, \quad 0<x<\infty,
$$

(where $\gamma=0.577 \cdots$ is Euler's constant) we obtain, since $E\left(x_{1}(s)\right)=\log (1 /(1-z))$,

$$
\begin{aligned}
x_{1}(s) & =(1-z) e^{-\gamma} \exp \left[\int_{0}^{x_{1}(s)} \frac{1-e^{-y}}{y} d y\right] \\
& =(1-z) e^{-\gamma}+O(1-z)^{2}, \quad z \rightarrow 1 .
\end{aligned}
$$

For the last expression we have used the bound

$$
0<\int_{0}^{x_{1}(s)} \frac{1-e^{-y}}{y} d y<x_{1}(s)<s
$$

given in the preceding paragraph.

Although we do not use it, the following expression for $x_{j}(s)$ may be of interest. We find $x_{j}(s)=\sum_{p=1}^{\infty} A_{p}(j) s^{p}$, convergent for all $s$, with the first few coefficients 


$$
\begin{aligned}
A_{1}(j) & =\exp \left[\Gamma^{\prime}(j) / \Gamma(j)\right]=\exp \left[-\gamma+\sum_{1}^{j-1}(1 / k)\right] \\
& \sim j-\frac{1}{2}+\frac{1}{24 j}+\frac{1}{48 j^{2}}+\cdots, \\
A_{2}(j) & =A_{1}(j)\left(A_{1}(j)-j+\frac{1}{2}\right) \\
& \sim \frac{1}{24}+\frac{0}{j}+\cdots, \\
A_{3}(j) & =\frac{A_{1}(j)}{4}\left\{\left[A_{1}(j)-j+\frac{1}{2}\right]\left[5 A_{1}(j)-3\left(j-\frac{1}{2}\right)\right]-\frac{1}{12}\right\} \\
& \sim 0-\frac{1}{960 j}+\cdots, \\
A_{4}(j) & \sim-\frac{1}{2880}+\cdots .
\end{aligned}
$$

We omit the proof.

4. The $r$ th longest cycle. According to (6), the $m$ th moment of the length of the $r$ th longest cycle under $P_{z}$ is

$E_{z}\left\{\left(L_{r}\right)^{m}\right\}=\sum_{j=1}^{\infty} j^{m} \int_{t_{j}(z)}^{t_{j+1}(z)} \frac{\left[t_{\infty}(z)-t\right]^{r-1}}{(r-1) !} \exp \left[-\left[t_{\infty}(z)-t\right]\right] d t, \quad m, r=1,2, \cdots$.

Under the change of variable $t_{\infty}(z)-t=E(x)$, this becomes

$$
E_{z}\left\{\left(L_{r}\right)^{m}\right\}=\sum_{j=1}^{\infty} j^{m} \int_{x_{j}(s)}^{x_{j+1}(s)} \frac{[E(x)]^{r-1}}{(r-1) !} e^{-E(x)} \frac{e^{-x}}{x} d x
$$

with $z=e^{-s}$ again and $x_{j}(s)$ given by (8). Now $x_{j}(s)<j s<x_{j+1}(s)$, so that the quantity $s^{m} E_{z}\left\{\left(L_{r}\right)^{m}\right\}$ is bounded above by $\sum_{j=1}^{\infty}\left[x_{j+1}(s)\right]^{m} \mu_{j}$ and below by $\sum_{j=1}^{\infty}\left[x_{j}(s)\right]^{m} \mu_{j}$, with

$$
\begin{aligned}
\mu_{j} & =\int_{x_{j}(s)}^{x_{j+1}(s)} d \mu(x), \\
d \mu(x) & =\frac{[E(x)]^{r-1}}{(r-1) !} e^{-E(x)} \frac{e^{-x}}{x} d x .
\end{aligned}
$$

These bounds are just the upper and lower Darboux sums for the Darboux-Stieltjes integral $\int_{x_{1}(s)}^{\infty} x^{m} d \mu(x)$ based on the mesh determined by $x_{j}(s), j=1,2, \cdots$. From $(j-1) s<x_{j}(s)<j s<x_{j+1}(s)<(j+1) s$ it follows that the mesh size vanishes as $s \rightarrow 0$. As $z \rightarrow 1$ then, we have $s=\log (1 / z) \rightarrow 0, s /(1-z) \rightarrow 1, x_{1}(s) \rightarrow 0$, and hence

$$
\lim _{z \rightarrow 1} \frac{(1-z)^{m}}{m !} E_{z}\left\{\left(L_{r}\right)^{m}\right\}=G_{r, m}, \quad \begin{aligned}
& m=0,1, \cdots, \\
& r=1,2, \cdots,
\end{aligned}
$$

where the constants $G_{r, m}$ are 


$$
G_{r, m}=\int_{0}^{\infty} \frac{x^{m-1}}{m !} \frac{[E(x)]^{r-1}}{(r-1) !} \exp [-E(x)-x] d x .
$$

In $\S 6$ we prove that $E_{n}\left\{\left(L_{r}\right)^{m}\right\}$ is monotone nondecreasing in $n$. If we apply the Karamata-Hardy-Littlewood Tauberian theorem [7, p. 143], [8, p. 203] to (11) we obtain

$$
\lim _{n \rightarrow \infty} E_{n}\left\{\left(\frac{L_{r}}{n}\right)^{m}\right\}=G_{r, m}, \quad \begin{aligned}
m & =0,1, \cdots, \\
r & =1,2, \cdots,
\end{aligned}
$$

for the limiting form of the moments of $L_{r} / n$. The case $m=1, r=1$ is the limit of Golomb mentioned in the Introduction:

$$
\left.\lim _{n \rightarrow \infty} E_{n}\left\{\frac{L_{1}}{n}\right\}=\int_{0}^{\infty} \exp \left[-x-\int_{x}^{\infty}\left(e^{-y} / y\right) d y\right] d x\right)
$$

The numbers $G_{r, m}$ have the property

$$
\lim _{r \rightarrow \infty}(m+1)^{r} G_{r, m}=\frac{e^{-m \gamma}}{m !}, \quad m=0,1, \cdots .
$$

We sketch the proof. The change of variable $E(x)=\tau$ in (12) gives

$$
G_{r, m}=\int_{0}^{\infty} \frac{[\xi(\tau)]^{m}}{m !} \frac{\tau^{r-1}}{(r-1) !} e^{-\tau} d \tau
$$

where $\xi(\tau)$ is the function inverse to $E(x): \xi(E(x))=x, 0<x<\infty$. For large $r$ the density $\tau^{r-1} e^{-\tau} /(r-1)$ ! is negligible if $\tau$ is not large. From the identity (9) we obtain easily $\xi(\tau) \sim e^{-\tau-\gamma}, \tau \rightarrow \infty$, for the asymptotic form of $\xi(\tau)$. We substitute this in the expression for $G_{r, m}$ and the result follows. (The argument can be made rigorous; we omit the details.)

For the limiting distribution of $L_{r} / n$ we seek a distribution $F_{r}(\xi), 0 \leqq \xi \leqq 1$, with the property $\int_{0}^{1} \xi^{m} d F_{r}(\xi)=G_{r, m}, m=0,1, \cdots$. If such an $F_{r}$ exists then

$$
\lim _{n \rightarrow \infty} P_{n}\left\{\left(\frac{L_{r}}{n}\right) \leqq \xi\right\}=F_{r}(\xi), \quad 0 \leqq \xi \leqq 1
$$

is obtained according to the method of moments [9, p. 27]. From (12) it is straightforward that if there is an $F_{r}$ such that

$$
\int_{0}^{1} e^{-y / \xi} d F_{r}(\xi)=\int_{y}^{\infty} \frac{[E(x)]^{r-1}}{(r-1) !} e^{-E(x)} \frac{e^{-x}}{x} d x
$$

$$
=\sum_{p=r}^{\infty} \frac{(-1)^{p-r}[E(y)]^{p}}{(r-1) !(p-r) ! p}, \quad 0<y<\infty
$$


Mrs. L. A. Needham of our organization has obtained the following numerical values for the first moments $G_{r, 1}$.

\begin{tabular}{|c|c|c|}
\hline$r$ & $G_{r, 1}$ & $2^{r} e^{\gamma} G_{r, 1}$ \\
\hline 1 & 0.62432997 & 2.2239538 \\
\hline 2 & 0.20958090 & 1.4931151 \\
\hline 3 & 0.08831609 & 1.2583788 \\
\hline 4 & 0.04034198 & 1.1496320 \\
\hline 5 & 0.01914548 & 1.0911837 \\
\hline 6 & 0.00927494 & 1.0572380 \\
\hline 7 & 0.00454696 & 1.0366046 \\
\hline 8 & 0.00224518 & 1.0236979 \\
\hline 9 & 0.00111357 & 1.0154706 \\
\hline 10 & 0.00055387 & 1.0101584 \\
\hline 11 & 0.00027599 & 1.0066977 \\
\hline 12 & 0.00013768 & 1.0044290 \\
\hline 13 & 0.00006874 & 1.0029350 \\
\hline 14 & 0.00003434 & 1.0019480 \\
\hline 15 & 0.00001716 & 1.0012943 \\
\hline 16 & 0.00000857 & 1.0008607 \\
\hline 17 & 0.00000429 & 1.0005727 \\
\hline 18 & 0.00000214 & 1.0003812 \\
\hline 19 & 0.00000107 & 1.0002538 \\
\hline 20 & 0.00000054 & 1.0001690 \\
\hline 21 & 0.00000027 & 1.0001125 \\
\hline 22 & 0.00000013 & 1.0000749 \\
\hline 23 & 0.00000007 & 1.0000498 \\
\hline sum) & 0.99 & \\
\hline
\end{tabular}

TADLE 1. $G_{r, 1}$ and $2^{r} e^{\gamma} G_{r .1}$ for $r=1(1) 23$.

then $F_{r}$ will have moments $G_{r, m}$. Now, $E(y)$ is the Laplace transform $E(y)$ $=\int_{0}^{\infty} e^{-y u} f(u) d u$ of

$$
\begin{aligned}
f(u) & =0, & & 0 \leqq u<1, \\
& =\frac{1}{u}, & & 1 \leqq u<\infty,
\end{aligned}
$$


so that the rightmost member of (15) is indeed a Laplace transform. Inverting, we obtain

$$
\begin{aligned}
1-F_{r}(\xi) & =\sum_{p=r}^{p<1 / \xi} \frac{(-1)^{p-r}}{(r-1) !(p-r) ! p} \int_{\substack{u_{1} \geqq 1 \\
u_{1}+\ldots+u_{p} \leqq 1 / \xi}} \cdots \int_{\substack{u_{p} \geqq 1 \\
u_{1}}} \frac{d u_{1}}{u_{1}} \cdots \frac{d u_{p}}{u_{p}}, \quad 0<\xi<\frac{1}{r} \\
& =0, \frac{1}{r} \leqq \xi<\infty
\end{aligned}
$$

for the limiting distribution of $L_{r} / n$. We observe that for given $r, 1-F_{r}(\xi)$ is the sum of $q-r+1$ elementary integrals on the interval $(q+1)^{-1}<\xi \leqq q^{-1}$, $q=r, r+1, \cdots$. When $r=1$ the above is Goncharov's formula for the limiting distribution of $L_{1} / n$ [2].

5. The $r$ th shortest cycle. As $z \rightarrow 1$, the endpoints $t_{j}(z)$ of our model move to limiting positions

$$
\begin{array}{rlrl}
t_{j}=\lim _{z \rightarrow 1} t_{j}(z) & =\sum_{1}^{j-1} \frac{1}{k}, & & j=2,3, \cdots, \\
& =0, & j=1,
\end{array}
$$

and $S_{r}$ has the $z$ limiting distribution

$$
\lim _{z \rightarrow 1} P_{z}\left\{S_{r}=j\right\}=\int_{t_{j}}^{t_{j+1}} \frac{t^{r-1}}{(r-1) !} e^{-t} d t, \quad j=1,2, \cdots .
$$

In $\S 6$ we will show that the Tauberian side condition

$$
\left|P_{n}\left\{S_{r}=j\right\}-P_{n-1}\left\{S_{r}=j\right\}\right| \leqq \frac{2}{n}, \quad n=1,2, \cdots,
$$

holds, so from (16) and the KHLT theorem we have

$$
\lim _{n \rightarrow \infty} P_{n}\left\{S_{r}=j\right\}=\int_{t_{j}}^{t_{j+1}} \frac{t^{r-1}}{(r-1) !} e^{-t} d t, \quad j=1,2, \cdots .
$$

The tail of this distribution is asymptotically

$$
\frac{e^{-\gamma}[\log j]^{r-1}}{(r-1) ! j^{2}}, \quad j \rightarrow \infty,
$$

using the well-known estimate

$$
t_{j}=\sum_{1}^{j-1} \frac{1}{k}=\log j+\gamma+O\left(\frac{1}{j}\right)
$$

We see that the limiting distribution of $S_{r}$ has infinite mean. 
For the moments of $S_{r}$ under $P_{z}$ we have from (5)

$$
E_{z}\left\{\left(S_{r}\right)^{m}\right\}=\sum_{j=1}^{\infty} j^{m} \int_{t_{j}(z)}^{t_{j+1}(z)} \frac{t^{r-1}}{(r-1) !} e^{-t} d t,
$$

and the change of variable $t_{\infty}(z)-t=E(x)$ gives

$E_{z}\left\{\left(S_{r}\right)^{m}\right\}=(1-z) \sum_{j=1}^{\infty} j^{m} \int_{x_{j}(s)}^{x_{j+1}(s)} \frac{\left[t_{\infty}(z)-E(x)\right]^{r-1}}{(r-1) !} e^{E(x)} \frac{e^{-x}}{x} d x, \quad m, r=1,2, \cdots$,

with $z=e^{-s}$ again and $x_{j}(s)$ as in (8).

From the bounds $(j-1) s<x_{j}(s)<j s<x_{j+1}(s)<(j+1) s$ obtained in $\S 3$ there follows

$$
\sum_{1}^{\infty}[(j-1) s]^{m} \Theta_{j}<D_{-}<\sum_{1}^{\infty}(j s)^{m} \Theta_{j}<D_{+}<\sum_{1}^{\infty}[(j+1) s]^{m} \Theta_{j}
$$

where

$$
\begin{aligned}
& D_{-}=\sum_{1}^{\infty}\left[x_{j}(s)\right]^{m} \Theta_{j}, \\
& D_{+}=\sum_{1}^{\infty}\left[x_{j+1}(s)\right]^{m} \Theta_{j}, \\
& \Theta_{j}=\int_{x_{j}(s)}^{x_{j+1}(s)} \frac{\left[t_{\infty}(z)-E(x)\right]^{r-1}}{(r-1) !} e^{E(x)} \frac{e^{-x}}{x} d x .
\end{aligned}
$$

This and the elementary inequalities

$$
\begin{aligned}
& (j+1)^{m} \leqq j^{m}+\left(2^{m}-1\right) j^{m-1} \\
& (j-1)^{m} \geqq j^{m}-m j^{m-1}, \quad j, m=1,2, \cdots,
\end{aligned}
$$

now give

$$
\text { (17) } \begin{aligned}
\frac{(1-z)^{m-1}}{m-1 !} E_{z}\left\{\left(S_{r}\right)^{m}\right\}= & \left(\frac{1-z}{s}\right)^{m} \int_{x_{1}(s)}^{\infty} \frac{x^{m-1}}{(m-1) !} \frac{\left[t_{\infty}(z)-E(x)\right]^{r-1}}{(r-1) !} \\
& \cdot \exp [E(x)-x] d x+\Delta(r, m ; z), \quad m, r=1,2, \cdots,
\end{aligned}
$$

with

$$
\begin{aligned}
|\Delta(r, m ; z)| & \leqq(1-z)-1 \cdot \frac{\left(2^{m}-1\right)}{m-1} \cdot \frac{(1-z)^{m-2}}{(m-2) !} E_{z}\left\{\left(S_{r}\right)^{m-1}\right\}, \quad m=2,3, \cdots \\
& \leqq 1, \quad m=1
\end{aligned}
$$

We proceed by induction on $m$. For $m=1$ we integrate by parts in (17): 


$$
\begin{aligned}
\int_{x_{1}(s)}^{\infty} & \frac{\left[t_{\infty}(z)-E(x)\right]^{r-1}}{(r-1) !} \exp [E(x)-x] d x \\
= & x_{1}(s) e^{E\left(x_{1}(s)\right)} \frac{\left[t_{\infty}(z)\right]^{r}}{r !} \\
& -\int_{x_{1}(s)}^{\infty}\left(1-e^{-x}\right) e^{E(x)} \frac{\left\{\left[t_{\infty}(z)-E(x)\right]^{r}-\left[t_{\infty}(z)\right]^{r}\right\}}{r !} d x,
\end{aligned}
$$

and separate the formal powers of $t_{\infty}(z)=\log (1 /(1-z))=E\left(x_{1}(s)\right)$ :

where

$$
E_{z}\left\{S_{r}\right\}=\sum_{p=0}^{r} \frac{1}{p !}\left[\log \frac{1}{1-z}\right]^{p} H(r-p ; z)+O(1)
$$

$$
\begin{aligned}
& H(0 ; z)=\frac{x_{1}(s)}{s} \\
& H(q ; z)=-\left(\frac{1-z}{s}\right) \int_{x_{1}(s)}^{\infty}\left(1-e^{-x}\right) e^{E(x)} \frac{[-E(x)]^{q}}{q !} d x, \quad q=1,2, \cdots .
\end{aligned}
$$

Using (9) and (10), we see that as $z \rightarrow 1$,

with

$$
H(q ; z)=H(q)\left[1+O\left((1-z)\left(\log \frac{1}{1-z}\right)^{q}\right)\right], \quad q=0,1, \cdots
$$

$$
\begin{aligned}
& H(0)=e^{-\gamma} \\
& H(q)=-\int_{0}^{\infty}\left(1-e^{-x}\right) e^{E(x)} \frac{[-E(x)]^{q}}{q !} d x, \quad q=1,2, \cdots,
\end{aligned}
$$

whence

(18) $E_{z}\left\{S_{r}\right\}=\sum_{p=1}^{r} \frac{H(r-p)}{p !}\left[\log \frac{1}{1-z}\right]^{p}+O(1), \quad z \rightarrow 1, \quad r=1,2, \cdots$.

For $m=2,3, \cdots$ we express (17) as

$$
\begin{aligned}
& \frac{(1-z)^{m-1}}{(m-1) !} E_{z}\left\{\left(S_{r}\right)^{m}\right\} \\
= & \sum_{p=0}^{r-1} \frac{1}{p !}\left[\log \frac{1}{1-z}\right]^{p} K(r-1-p, m ; z)+\Delta(r, m ; z), \quad m=2,3, \cdots, \quad r=1,2, \cdots,
\end{aligned}
$$

where the coefficients are

$$
\begin{array}{r}
K(q, m ; z)=\left(\frac{1-z}{s}\right)^{m} \int_{x_{1}(s)}^{\infty} \frac{x^{m-1}}{(m-1) !} \frac{[-E(x)]^{q}}{q !} \exp [E(x)-x] d x, \\
m=2,3, \cdots, \quad q=0,1, \cdots .
\end{array}
$$


Using (9) and (10) again, we find

$$
\begin{array}{r}
\int_{0}^{x^{1}(s)} x^{m-1}[E(x)]^{q} \exp [E(x)-x] d x=O\left((1-z)^{m-1}\left(\log \frac{1}{1-z}\right)^{q}\right), \\
m=2,3, \cdots, \quad q=0,1, \cdots
\end{array}
$$

This and induction on $m$ in (17) give

$$
\begin{aligned}
& \frac{(1-z)^{m-1}}{(m-1) !} E_{z}\left\{\left(S_{r}\right)^{m}\right\} \\
& =\sum_{p=0}^{r-1} \frac{K(r-1-p, m)}{p !}\left[\log \frac{1}{1-z}\right]^{p}+O\left((1-z)\left(\log \frac{1}{1-z}\right)^{r}\right), \\
& z \rightarrow 1, \quad m=2,3, \cdots, \quad r=1,2, \cdots,
\end{aligned}
$$

with coefficients

$$
K(q, m)=\int_{0}^{\infty} \frac{x^{m-1}}{(m-1) !} \frac{[-E(x)]^{q}}{q !} \exp [E(x)-x] d x, q=0,1, \cdots, \quad m=2,3, \cdots .
$$

Formal inversion of (18) gives, for $n \rightarrow \infty$,

(20) $E_{n}\left\{S_{r}\right\}$

$$
\begin{array}{r}
=\left.\frac{1}{r !}\left(\frac{\partial}{\partial \xi}\right)^{r} \frac{n^{\xi}}{\Gamma(\xi+1)}\left\{e^{-\gamma}-\int_{0}^{\infty}\left(1-e^{-x}\right) e^{E(x)}\left[e^{-\xi E(x)}-1\right] d x\right\}\right|_{\xi=0}+O(1), \\
r=1,2, \cdots,
\end{array}
$$

while (19) gives similarly

(21) $E_{n}\left\{\left(S_{r}\right)^{m}\right\}$

$$
\begin{aligned}
=\frac{n^{m-1}}{(r-1) !}\left(\frac{\partial}{\partial \xi}\right)^{r-1} \frac{n^{\xi}}{\Gamma(m+\xi)} \int_{0}^{\infty} & \left.x^{m-1} \exp [(1-\xi) E(x)-x] d x\right|_{\xi=0} \\
& +O\left(n^{m-2}(\log n)^{r}\right), \quad m=2,3, \cdots, \quad r=1,2, \cdots .
\end{aligned}
$$

However, the authors can prove only that the leading term is correct in (20)-(21), viz.,

$$
\begin{gathered}
\lim _{n \rightarrow \infty} \frac{E_{n}\left\{S_{r}\right\}}{(\log n)^{r}}=\frac{e^{-\gamma}}{r !}, \quad r=1,2, \cdots, \\
\lim _{n \rightarrow \infty} \frac{E_{n}\left\{\left(S_{r}\right)^{m}\right\}}{n^{m-1}(\log n)^{r-1}}=\frac{1}{(r-1) !} \int_{0}^{\infty} \frac{x^{m-1}}{(m-1) !} \exp [E(x)-x] d x, \\
m=2,3, \cdots, \quad r=1,2, \cdots,
\end{gathered}
$$

as follows.

First, we apply the KHLT theorem with the side condition $E_{n}\left\{\left(S_{r}\right)^{m}\right\} \geqq 0$ in (18)-(19) to get 


$$
\begin{aligned}
& \sum_{n=0}^{N-1} E_{n}\left\{S_{r}\right\}=\frac{H(0)}{r !} N(\log N)^{r}[1+o(1)], \quad N \rightarrow \infty, \quad r=1,2, \cdots, \\
& \sum_{n=0}^{N-1} E_{n}\left\{\left(S_{r}\right)^{m}\right\}=\frac{K(0, m)}{(r-1) !} \frac{N^{m}(\log N)^{r-1}}{m}[1+o(1)], \quad N \rightarrow \infty, \\
& m=2,3, \cdots, \quad r=1,2, \cdots,
\end{aligned}
$$

for the $(C, 1)$ asymptotics of $E_{n}\left\{\left(S_{r}\right)^{m}\right\}$.

In $\S 6$ we prove the relation

$$
\begin{aligned}
& E_{n}\left\{\left(S_{r}\right)^{m}\right\}-E_{n-1}\left\{\left(S_{r}\right)^{m}\right\} \\
& \leqq \frac{1}{n} E_{n-1}\left\{\left(S_{r-1}\right)^{m}-\left(S_{r}\right)^{m}\right\}+\frac{\left(2^{m}-1\right) r}{n} E_{n-1}\left\{\left(S_{r}\right)^{m}\right\}, m, r, n=1,2, \cdots,
\end{aligned}
$$

where $S_{0}=1$. We multiply this by $n$, add $\left.E_{n-1}\left\{S_{r}\right)^{m}\right\}$ to both sides, and have

$$
n E_{n}\left\{\left(S_{r}\right)^{m}\right\}-(n-1) E_{n-1}\left\{\left(S_{r}\right)^{m}\right\} \leqq E_{n-1}\left\{\left(S_{r-1}\right)^{m}\right\}+\left(2^{m}-1\right) r E_{n-1}\left\{\left(S_{r}\right)^{m}\right\} .
$$

Now add these inequalities for $n=1, \cdots, N$. The left-hand members telescope and the right-hand sum may be bounded by using (23); we find, for $r=1,2, \cdots$, and all large $n$,

$$
\begin{aligned}
E_{n}\left\{\left(S_{r}\right)^{m}\right\} & \leqq C^{\prime}(\log n)^{r}, & & m=1, \\
& \leqq C^{\prime} n^{m-1}(\log n)^{r-1}, & & m=2,3, \cdots,
\end{aligned}
$$

with appropriate constants $0<C^{\prime}<\infty$ (depending on $m$ and $r$, of course). We substitute these bounds in the right-hand side of (24). There results

$$
\begin{aligned}
E_{n}\left\{\left(S_{r}\right)^{m}\right\} & -E_{n-1}\left\{\left(S_{r}\right)^{m}\right\} & & \\
& \leqq C n^{-1}(\log n)^{r}, & & m=1, \\
& \leqq C n^{m-2}(\log n)^{r-1}, & & m=2,3, \cdots,
\end{aligned}
$$

for some constants $0<C<\infty$, and we now have Tauberian side conditions for (18)-(19) which serve to prove (22).

6. The Tauberian side conditions. Let us now obtain the various bounds on quantitities $E_{n}\{\Phi\}-E_{n-1}\{\Phi\}$ which we used in $\S \S 4$ and 5.

For each $n=1,2, \cdots$, we define a mapping $\theta: \mathscr{S}_{n} \rightarrow \mathscr{S}_{n-1}$ which preserves the probability structure, as follows. We regard the elements of $\mathscr{S}_{n}$ as the permutation operators on $n$ distinguishable objects occupying places numbered $1, \cdots, n$; for definiteness let us agree that " $p \rightarrow q$ " means "the object in place $p$ moves to place $q$ " (and not "object $\mathrm{p}$ where it stands turns into object $q$ ".) If $\pi \in \mathscr{S}_{n}$ is given, write $\pi$ in the usual way as a product of disjoint cycles. Erase symbol $n$ in the cycle expression for $\pi$; the result is the cycle expression for $\theta(\pi) \in \mathscr{S}_{n-1}$. In more 
detail, let $\pi \in \mathscr{S}_{n}$ make the moves $p \rightarrow n \rightarrow q$ as far as place $n$ is concerned. Either (i) $p=n=q$, in which case $\theta(\pi)$ acts on the places different from $n$ the same way as $\pi$, or (ii) $p \neq n$ and $q \neq n$, in which case $\theta(\pi)$ makes the move $p \rightarrow q$ and agrees with $\pi$ otherwise. One sees that the $n$ ! values $\theta(\pi), \pi \in \mathscr{S}_{n}$, consist of $n$ copies each of the elements of $\mathscr{S}_{n-1}$. If $\pi^{\prime} \in \mathscr{S}_{n-1}$ is given, we denote by $\left(\pi^{\prime}, p\right), p=1, \cdots, n$ the $n$ distinct elements of $\theta^{-1}\left(\pi^{\prime}\right) \subset \mathscr{S}_{n} ; p$ is to be regarded as the variable appearing in the above description of $\theta$. When each element of $\mathscr{S}_{n}$ has probability $1 / n$ ! the mapping $\theta: \mathscr{S}_{n} \rightarrow \mathscr{S}_{n-1}$ induces the distribution $P_{n}\left\{\theta^{-1}\left(\pi^{\prime}\right)\right\}=n / n$ ! $=1 /(n-1) !=P_{n-1}\left\{\pi^{\prime}\right\}, \pi^{\prime} \in \mathscr{S}_{n-1}$, as desired.

If $\Phi$ is a functional defined on every $\mathscr{S}_{n}$, the difference $E_{n}\{\Phi\}-E_{n-1}\{\Phi\}$ reduces to the average pointwise difference

$$
\begin{aligned}
& E_{n}\{\Phi\}-E_{n-1}\{\Phi\} \\
& =\frac{1}{n !} \sum_{\pi \in \mathscr{S}_{n}}[\Phi(\pi)-\Phi(0(\pi))] \\
& =\frac{1}{(n-1) !} \sum_{\pi \in \mathscr{P}_{n-1}} \frac{1}{n} \sum_{p=1}^{n}\left[\Phi\left(\left(\pi^{\prime}, p\right)\right)-\Phi\left(\pi^{\prime}\right)\right], \quad n=1,2, \cdots ;
\end{aligned}
$$

both forms are useful.

Let us consider first the $r$ th longest cycle. Suppose for given $\pi \in \mathscr{S}_{n}$ the values of $L_{r}, r=1,2, \cdots$ are $L_{1}(\pi) \geqq L_{2}(\pi) \cdots \geqq 0$. For $\theta(\pi)$ the cycle lengths are the same numbers except for one number, wich is less by 1 than before (but not negative). If the new list is rearranged in descending order the $r$ th number is the same as before except when a cycle of length $L_{r}(\pi)$ has been shortened and $L_{r}(\pi)$ $>L_{r+1}(\pi)$ holds, in which event there obtains $L_{r}(\theta(\pi))=L_{r}(\pi)-1$. It follows from (25) that

$$
\begin{aligned}
E_{n} & \left\{\left(L_{r}\right)^{m}\right\}-E_{n-1}\left\{\left(L_{r}\right)^{m}\right\} \\
& =\sum_{j=1}^{n}\left[j^{m}-(j-1)^{m}\right] P_{n}\left\{L_{r}=j>L_{r+1}\right.
\end{aligned}
$$

\& symbol $n$ appears in a cycle of length $j\}, n, r, m=1,2, \cdots$.

The conditional probability that symbol $n$ appears in a cycle of length $j$, given the cycle class, is $j \alpha_{j} / n$, so

$$
\begin{aligned}
E_{n}\left\{\left(L_{r}\right)^{m}\right\}-E_{n-1}\left\{\left(L_{r}\right)^{m}\right\} \\
\quad=\sum_{j-1}^{n}\left[j^{m}-(j-1)^{m}\right] E_{n}\left\{\frac{j a_{j}}{n} \chi\left\{L_{r}=j>L_{r+1}\right\}\right\}
\end{aligned}
$$

where $\chi\{\}$ denotes the characteristic function of event \{\} . It is obvious that the above difference is nonnegative; this is all that is needed in $\S 4$. The following 
refinement is interesting, however. The event $\left\{L_{r}=j>L_{r+1}\right\}$ in $\mathscr{S}_{n}$ is just the event $\left\{\alpha_{j}>0, \alpha_{j}+\cdots+\alpha_{n}=r\right\}$, so that $1 \leqq \alpha_{j} \leqq r$ for this event. Thus

$$
\begin{aligned}
E_{n}\left\{\left(L_{r}\right)^{m}\right\} & -E_{n-1}\left\{\left(L_{r}\right)^{m}\right\} \\
& \leqq \sum_{j=1}^{n} m j^{m-1}(r j / n) P_{n}\left\{L_{r}=j\right\} \\
& =\frac{m r}{n} E_{n}\left\{\left(L_{r}\right)^{m}\right\}, \quad n, r, m=1,2, \cdots .
\end{aligned}
$$

With $m=1, r=1$ this yields Golomb's results

$$
\frac{E_{n-1}\left(L_{1}\right)}{n-1} \geqq \frac{E_{n}\left(L_{1}\right)}{n}, \quad n=2,3, \cdots .
$$

If we work instead from $n-1$ to $n$, we obtain a different result. Suppose the values of $L_{r}, r=1,2, \cdots$, for $\pi^{\prime} \in \mathscr{S}_{n-1}$ are $L_{1}\left(\pi^{\prime}\right) \geqq L_{2}\left(\pi^{\prime}\right) \geqq \cdots \geqq 0$. In $\left(\pi^{\prime}, p\right)$ the cycle lengths are the same numbers except for one number, which is greater by 1 than before. If the new list is rearranged in descending order, the $r$ th number is the same as before except when a cycle of length $L_{r}\left(\pi^{\prime}\right)$ has been lengthened and $L_{r-1}\left(\pi^{\prime}\right)>L_{r}\left(\pi^{\prime}\right)$ holds (put $L_{0}=\infty$ ), in which event there obtains $L_{r}\left(\left(\pi^{\prime}, p\right)\right)=L_{r}\left(\pi^{\prime}\right)+1$. There follows

$$
\begin{aligned}
E_{n}\left\{\left(L_{r}\right)^{m}\right\}- & E_{n-1}\left\{\left(L_{r}\right)^{m}\right\} \\
= & \frac{1}{n} P_{n-1}\left\{L_{r}=0<L_{r-1}\right\} \\
& \quad+\frac{1}{n} \sum_{j=1}^{n-1}\left[(j+1)^{m}-j^{m}\right] E_{n-1}\left\{j \alpha_{j} \chi\left\{L_{r}=j<L_{r-1}\right\}\right\} \\
\geqq & \frac{m}{n} \sum_{j=1}^{n-1} j^{m} P_{n-1}\left\{L_{r}=j<L_{r-1}\right\}, \quad m, r, n=1,2, \cdots
\end{aligned}
$$

For $m=1, r=1$ this is Golomb's result

$$
\frac{E_{n-1}\left\{L_{1}\right\}}{(n-1)+1} \leqq \frac{E_{n}\left\{L_{1}\right\}}{n+1}, \quad n=1,2, \cdots .
$$

The authors have used the above to prove the result $E_{n}\left\{L_{1}\right\}=\left(n+\frac{1}{2}\right) G_{1,1}+o(1)$ conjectured by Golomb on the basis of numerical computations. We omit the details. Presumably there is a hierarchy of successively more delicate side conditions needed to establish the lower order terms in $E_{n}\left\{\left(L_{r}\right)^{m}\right\}$.

Consider now the length of the $r$ th shortest cycle. Let

$$
S_{1}(\pi) \leqq S_{2}(\pi) \leqq \cdots \leqq S_{\sigma}(\pi), 0,0, \cdots
$$

be the values of $S_{r}, r=1,2, \cdots$ for $\pi \in \mathscr{S}_{n}$. The cycle lengths of $\theta(\pi)$ are the same 
numbers except for one number, which is less by 1 than before (but not negative). If the first $\sigma$ members of the new list are rearranged in ascending order and the initial 0 (if any) is deleted, the $r$ th number in the new list is the same as in the old except possibly when (i) symbol $n$ appears in $\pi$ in a cycle of length 1 ; in this event $S_{r}(\theta(\pi))=S_{r+1}(\pi)$, (ii) symbol $n$ appears in $\pi$ in a cycle of length $S_{r}(\pi)>1$ and $S_{r-1}(\pi)<S_{r}(\pi)$ holds (put $S_{0}=1$ ); in this event $S_{r}(\theta(\pi))=S_{r}(\pi)-1$. For the distribution of $S_{r}$ we obtain from (25)

$$
\begin{aligned}
& \left|P_{n}\left\{S_{r}=j\right\}-P_{n-1}\left\{S_{r}=j\right\}\right| \\
& =\left|E_{n}\left\{\chi\left\{S_{r}=j\right\}\right\}-E_{n-1}\left\{\chi\left\{S_{r}=j\right\}\right\}\right| \\
& =\mid E_{n}\left\{\frac{\alpha_{1}}{n} \chi\left\{S_{r}=j \neq S_{r+1}\right\}-\frac{\alpha_{1}}{n} \chi\left\{S_{r} \neq j=S_{r+1}\right\}\right\} \\
& \quad+E_{n}\left\{\frac{j \alpha_{j}}{n} \chi\left\{S_{r-1}<j=S_{r}\right\}-\frac{(j+1) \alpha_{j+1}}{n} \chi\left\{S_{r-1}<j+1=S_{r}\right\}\right\} \mid \\
& \leqq \\
& \frac{2}{n}, \quad n=1,2, \cdots, \quad j=0,1, \cdots,
\end{aligned}
$$

since $E_{n}\left\{j \alpha_{j}\right\}=1$ for $1 \leqq j \leqq n$ and $=0$ for $n<j$. The bound above is the Tauberian side condition used in $\S 5$ to obtain the limit of $P_{n}\left\{S_{r}=j\right\}$.

For the moments of $S_{r}$ we obtain by similar arguments

$$
\begin{aligned}
E_{n}\left\{\left(S_{r}\right)^{m}\right\}- & E_{n-1}\left\{\left(S_{r}\right)^{m}\right\} \\
= & E_{n}\left\{\frac{\alpha_{1}}{n}\left[\left(S_{r}\right)^{m}-\left(S_{r+1}\right)^{m}\right]\right\} \\
& +\sum_{j=2}^{n}\left[j^{m}-(j-1)^{m}\right] E_{n}\left\{\frac{j \alpha_{j}}{n} \chi\left\{S_{r-1}<j=S_{r}\right\}\right\} \\
= & \frac{1}{n} E_{n-1}\left\{\left(S_{r-1}\right)^{m}-\left(S_{r}\right)^{m}\right\} \\
& +\frac{1}{n} \sum_{j=1}^{n-1}\left[(j+1)^{m}-j^{m}\right] E_{n-1}\left\{j \alpha_{j} \chi\left\{S_{r}=j \neq S_{r+1}\right\}\right\}, m, r, n=1,2, \cdots,
\end{aligned}
$$

where, again, $S_{0}=1$. Let us use the second form. The event $\left\{S_{r}=j \neq S_{r+1}\right\}$ is just the event $\left\{\alpha_{j}>0, \alpha_{1}+\cdots+\alpha_{j}=r\right\}$, so $1 \leqq \alpha_{j} \leqq r$ for this event. Thus

$$
\begin{aligned}
E_{n}\left\{\left(S_{r}\right)^{m}\right\} & -E_{n-1}\left\{\left(S_{r}\right)^{m}\right\} \\
\leqq & \frac{1}{n} E_{n-1}\left\{\left(S_{r-1}\right)^{m}-\left(S_{r}\right)^{m}\right\} \\
& \quad+\frac{1}{n} \sum_{j=1}^{n-1}\left(2^{m}-1\right) j^{m-1}(j r) P_{n-1}\left\{S_{r}=j \neq S_{r+1}\right\} \\
\leqq & \frac{1}{n} E_{n-1}\left\{\left(S_{r-1}\right)^{m}-\left(S_{r}\right)^{m}\right\}+\frac{\left(2^{m}-1\right) r}{n} E_{n-1}\left\{\left(S_{r}\right)^{m}\right\}, \quad m, r, n=1,2, \cdots,
\end{aligned}
$$

and this is the inequality used in $\$ 5$. 


\section{REFERENCES}

1. John Riordan, An introduction to combinatorial analysis, Wiley, New York, 1958.

2. V. Goncharov, Sur la distribution des cycles dans les permutations, C. R. (Doklady) Acad. Sci. URSS (N.S) 35 (1942), 267-269.

3. - Du domaine d'analyse combinatoire, Bull. Acad. Sci. USSR Ser. Mat. (Izv. Akad. Nauk SSSR) 8 (1944), 3-48; Amer. Math. Soc. Transl. (2) 19 (1962), 1-46.

4. S. W. Golomb, L. R. Welch and R. M. Goldstein, Cycles from nonlinear shift registers Progress Rep. No. 20-389, Jet Propulsion Laboratory, California Institute of Technology, Pasadena, Calif., 1959.

5. S. W. Golomb, Random permutations, Bull. Amer. Math. Soc. 70 (1964), 747.

6. E. T. Whittaker and G. N. Watson, Modern analysis, Cambridge Univ. Press, New York, 1948.

7. N. G. de Bruijn, Asymptotic methods in analysis, North-Holland, Amsterdam, 1961.

8. G. Doetsch, Laplace-transformation, reprint, Dover, New York, 1943.

9. Mark Kac, Probability and related topics in physical sciences, Interscience, New York, 1959.

Bell Telephone Laboratories, INCOR PORATED, MurRay Hill, New Jersey 